\title{
One-Dimensional Modelling of Developable Elastic Strips by Geometric Constraints and their Link to Surface Isometry
}

\author{
$\underline{\text { Benjamin Bauer }^{1,2},}$ Michael Roller $^{1}$, Joachim Linn $^{1}$, Bernd Simeon $^{2}$ \\ ${ }^{1}$ Mathematics for the Digital Factory \\ Fraunhofer Institute for Industrial Mathematics (ITWM) \\ Fraunhofer Platz 1, 67663 Kaiserslautern, Germany \\ [benjamin.bauer, michael.roller, joachim.linn]@itwm.fraunhofer.de \\ ${ }^{2}$ Felix-Klein-Zentrum for Mathematics \\ TU Kaiserslautern \\ Erwin-Schrödinger-Straße 52, 67663 Kaiserslautern, Germany \\ simeon@mathematik.uni-kl.de
}

\begin{abstract}
The goal of this paper is to introduce a kinematical reduction for the structural model of Kirchhoff-Love shells with developable base surfaces. The dimensional reduction to a curve and a vector field along it decreases the involved number of degrees of freedom. Local coordinates in form of a relatively parallel frame allow us to simplify the geometric constraints occurring in the model and prevent instabilities caused by points or segments of zero curvature. The core of this work is to prove equivalence of these requirements and the isometry of the transformation. Subsequently, we derive the one-dimensional bending energy functional for rectangular strips. In order to compute the equilibrium state of a static shell, we minimise a penalised version of this functional over the finitely many degrees of freedom stemming from an isogeometric discretisation. Several example strips clamped at both ends illustrate the feasibility of this approach.
\end{abstract}

Keywords: developable surfaces, Bishop frame, Kirchhoff-Love shells, isogeometric discretization, energy method.

\section{INTRODUCTION}

Thin-walled structural parts, denoted as shell structures in Computational Mechanics, frequently appear in engineering designs due to their special mechanical properties (cf. [1, sec. 1.1]). These are mainly determined by their geometric characteristics: Two parts, manufactured from the same material and subject to the same external loads, can be designed geometrically to react either very stiff or easily deformable.

Classical shell theories [2] aim at reducing both involved degrees of freedom and numerical costs by modelling sheet-like objects via their centre surface. Developable shell structures may be flattened by bending deformations, without any changes in the surface metric that imply high membrane stresses. Therefore, such surface geometries are good candidates for applications which demand highly flexible parts.

Flexible flat cables (FFCs) are prototypical examples of such structures and important components in computer hardware or consumer electronics products. Large spatial deformations of FFCs occur e.g. during the (dis-)assembly of such products. Their simulation is an essential part of a digitalized product development, as well as digital product lifecycle management.

Within the last century, several approaches to specialise Kirchhoff-Love type shell models for developable surfaces occurred [3]. Sadowsky [4] and Wunderlich [5] studied the bending energy of a narrow Kirchhoff-Love strip and integrated it along the direction of zero curvature, thereby 
reducing it to the length dimension only. Starostin and van der Heijden [6], as well as Dias and Audoly [7] recently proposed one-dimensional models for ribbons.

In our work we take first steps to investigate the suitability of specialized ruled surface shell models of this type as computational models for applications as sketched above for FFC. In section 2 , we consider the geometry of developable surfaces and justify geometric constraints that model isometric deformations. This generalises the approach proposed in [6] to base curves that possess arbitrary geodesic curvature and may exhibit points or segments of vanishing total curvature. Furthermore, we inspect surface singularities occurring from self-penetration of matter closer. A relatively parallel frame [8] (in literature also rotation minimising frame, parallel transported frame or Bishop frame) allows to decompose the director of the developable and thereby ruled surface. Section 3 proves the equivalence of our geometric model and vanishing of the surface membrane energy. Thus, the stored energy function of the transformations inspected consists only of the bending energy. We analytically integrate this energy over the second surface parameter and end up with a result similar to [5] in section 4. Section 5 explains the numerical treatment of the optimisation process: we address the non-linear constraints by a penalty method and employ an interior point method [9] to compute the stable equilibrium state. Finally, we display and discuss our results in section 6 .

\section{GEOMETRIC MODEL}

Every developable surface is ruled, that means it can be represented by a regular base curve (also called directrix) $\boldsymbol{\gamma}$ and a director vector field $\mathbf{d}$ in the form

$$
\phi:(u, v) \mapsto \boldsymbol{\gamma}(u)+v \mathbf{d}(u) .
$$

This representation scheme is illustrated in Figure 1. Conversely, a ruled surface is developable if and only if the determinant of curve tangent $\mathbf{t}$, director and director derivative $\operatorname{det}[\mathbf{t}, \mathbf{d}, \dot{\mathbf{d}}]$ vanishes everywhere along the base curve [10, chap. 5.5]. The dotted derivative stresses that $u$ is not necessarily an arc-length parameter, following the convention of [10], whereas a prime as in $\boldsymbol{\gamma}^{\prime}$ shall always denote derivatives with respect to the arc-length parameter $s$ of $\boldsymbol{\gamma}$.

We regard strips that possess dedicated (local) length, width and thickness dimensions. This means, we may assume $\gamma$ to match the centre-line of $\phi$ such that the width, defined as cross section in the normal plane to $\mathbf{t}$, is constantly $2 w$. Similarly, we define the thickness normal to $\boldsymbol{\phi}$ and assume it to constantly be $2 t$. The capital letter $L$ shall denote the length of $\boldsymbol{\gamma}$ and emphasize the geometric characteristics $L \gg w \gg t$ of the strip.

Developable surfaces may exhibit singularities in form of neighboured rulings intersecting each other as depicted in Figure 2, which may be seen as a violation of the impenetrability of matter. All these intersection points form an (in general not continuous) curve, called edge of regression (cf. [10, chap. 5.1]). Characteristically, the surface normal vanishes in these singular points $\left(u^{*}, v^{*}\right)$ such that

$$
\operatorname{det} \mathbf{A}\left(u^{*}, v^{*}\right)=\left\|\partial_{u} \boldsymbol{\phi}\left(u^{*}, v^{*}\right) \times \partial_{\nu} \boldsymbol{\phi}\left(u^{*}, v^{*}\right)\right\|^{2}=0,
$$

where $\boldsymbol{A}:(u, v) \mapsto\left[\partial_{\alpha} \boldsymbol{\phi}(u, v) \cdot \partial_{\beta} \boldsymbol{\phi}(u, v)\right]_{\alpha, \beta \in\{u, v\}}$ is the metric tensor and $\partial_{u} \boldsymbol{\phi}, \partial_{v} \boldsymbol{\phi}$ denote the tangent vectors of the surface in $\phi(u, v)$ along the isoparametric lines (constant $v$ or $u$, respectively).

Note that if director and curve tangent are parallel, the surface normal reads $v(\dot{\mathbf{d}} \times \mathbf{d})$. Hence, then there is a singularity lying on $\boldsymbol{\gamma}$. Thus, for regular surfaces, $\mathbf{t}$ and $\mathbf{d}$ need to be linearly independent. Furthermore, without loss of generality, we may scale $\mathbf{d}$ such that its component normal to $\boldsymbol{\gamma}$ is of unit length, i.e. $\|\mathbf{d}-(\mathbf{d} \cdot \mathbf{t}) \mathbf{t}\| \equiv 1$. The symbol $\equiv$ means pointwise equality for all parameters $u$. With this, the parameter domain of $\phi$ becomes rectangular and we may write $\boldsymbol{\phi}:[0, L] \times[-w, w] \rightarrow \mathbb{R}^{3}$. 


\subsection{Local Frame Coordinates}

In order to rigorously define the curvature terms in the subsequent paragraphs, we will assume the necessary smoothness $\boldsymbol{\gamma} \in \mathscr{C}^{2}$ and $\mathbf{d} \in \mathscr{C}^{1}$ throughout the whole paper, where $\mathscr{C}^{k}$ denotes the space of k-times continuously differentiable functions. This provides existence [11] of a relatively parallel frame $\left(\mathbf{t}, \mathbf{m}_{1}, \mathbf{m}_{2}\right)$ along $\boldsymbol{\gamma}$ with generalised Frenet equations

$$
\frac{\mathrm{d}}{\mathrm{d} u}\left[\begin{array}{c}
\mathbf{t} \\
\mathbf{m}_{1} \\
\mathbf{m}_{2}
\end{array}\right] \equiv\left[\begin{array}{lll} 
& k_{1} & k_{2} \\
-k_{1} & & \\
-k_{2} &
\end{array}\right]\left[\begin{array}{c}
\mathbf{t} \\
\mathbf{m}_{1} \\
\mathbf{m}_{2}
\end{array}\right]
$$

and enables us to decompose the director as

$$
\mathbf{d} \equiv d_{0} \mathbf{t}+d_{1} \mathbf{m}_{1}+d_{2} \mathbf{m}_{2}
$$

with coordinate functions $d_{0}, d_{1}, d_{2}$ with respect to the relatively parallel frame.

Using the curvature components $k_{1}, k_{2}$ of the frame, we may write

$$
\operatorname{det}[\mathbf{t}, \mathbf{d}, \dot{\mathbf{d}}] \equiv d_{1} \underbrace{\left(\dot{d}_{2}+d_{0} k_{2}\|\dot{\gamma}\|\right)}_{\equiv: \pi_{G}^{(2)}}-d_{2} \underbrace{\left(\dot{d}_{1}+d_{0} k_{1}\|\dot{\gamma}\|\right)}_{\equiv: \pi_{G}^{(1)}} .
$$

As $\operatorname{det}[\mathbf{t}, \mathbf{d}, \dot{\mathbf{d}}] \equiv 0$, the tangent plane of $\boldsymbol{\phi}$ is spanned by $\mathbf{t}$ and $\mathbf{d}$. Then the unit normal vector of $\boldsymbol{\phi}$ reads $\boldsymbol{\eta}(\cdot, v) \equiv \mathbf{t} \times \mathbf{d} \equiv-d_{2} \mathbf{m}_{1}+d_{1} \mathbf{m}_{2}$ independently of $v \in[-w, w]$.

\subsection{Rectangular Strips and Rectifying Developables}

In the special case of rectangular strips, the surface $\phi$ matches the rectifying developable (RD) of its centre-line $\boldsymbol{\gamma}$, which is the envelope of planes spanned by tangent and Frenet binormal $\mathbf{b}$. The model of [6] exploits this representation such that the director reads $\mathbf{d} \equiv \frac{\tau}{\kappa} \mathbf{t}+\mathbf{b}$, where $\kappa$ and $\tau$ denote the Frenet curvature and torsion, respectively.

Since the definition of the RD requires an existing Frenet frame, we generalise this concept to curves which are twice continuously differentiable. For this, we utilise three characteristic properties of the RD: $\phi$ is developable, $\gamma$ is a geodesic on $\phi$ and the curve tangent and director are pointwise linear independent [12, Proposition 4.1]. Note that we already treated the third condition above regarding regularity and that a geodesic line is characterised by having zero geodesic curvature

$$
\kappa_{g} \equiv \frac{\operatorname{det}[\dot{\boldsymbol{\gamma}}, \ddot{\boldsymbol{\gamma}}, \boldsymbol{\eta}]}{\|\dot{\boldsymbol{\gamma}}\|^{3}} \equiv k_{1} d_{1}+k_{2} d_{2}
$$

everywhere [13].

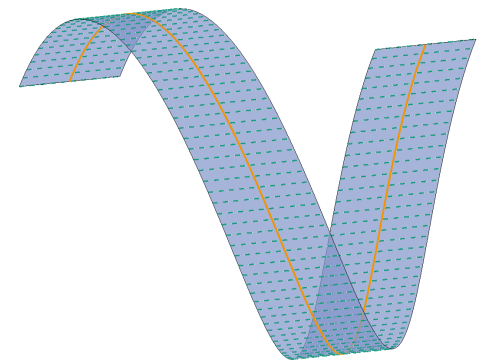

Figure 1: Representation of a helicoidal strip as ruled surface with orange base curve and greenly dashed rulings.

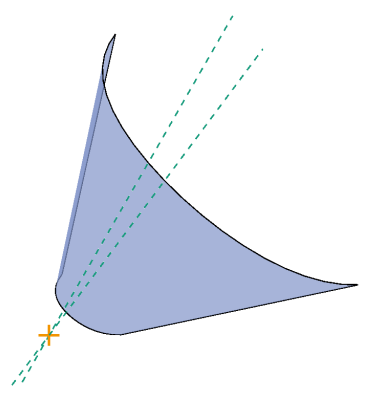

Figure 2: Intersection of a pair of neighboured rulings in an orange point outside of the strip. 
Thus, we get a system of geometric constraints

$$
\operatorname{det}[\mathbf{t}, \mathbf{d}, \dot{\mathbf{d}}] \equiv 0, \quad \kappa_{g} \equiv 0, \quad\|\mathbf{d}-(\mathbf{d} \cdot \mathbf{t}) \mathbf{t}\| \equiv 1 .
$$

If a ruled surface $\boldsymbol{\phi}$ satisfies these conditions, we call it generalised rectifying developable (GRD). Furthermore, the edge of regression restricts the surface width via the regularity condition (2) to the well-known [6, sec. 2.1]

$$
w<\min _{s \in[0, L]}\left|v^{*}(s)\right|=\frac{1}{\max _{s \in[0, L]}\left|\dot{d}_{0}(s)\right|},
$$

or vice versa $\left|\dot{d}_{0}\right|<\frac{1}{w}$ globally on $[0, L]$.

\subsection{Generalisation to Curved Bands}

Next, we follow the spirit of [7] and admit a priori curved directrix in the reference configuration. This means, we relax the requirement of $\boldsymbol{\gamma}$ being a geodesic and simply require the base curve to maintain its geodesic curvature. Note that this is obviously necessary concerning isometric transformations.

Consider the partial derivatives of $\boldsymbol{\phi}$ which define a unique normal vector if and only if their cross-product

$$
\left\|\boldsymbol{\phi}_{u} \times \boldsymbol{\phi}_{v}\right\| \equiv\|\dot{\boldsymbol{\gamma}}\|+v \dot{d}_{0}-v \kappa_{g}\|\dot{\boldsymbol{\gamma}}\|-d_{0} \kappa_{g}\|\dot{\boldsymbol{\gamma}}\|
$$

does not vanish. This yields for the critical value $v^{*}$

$$
v^{*} \equiv \frac{\|\dot{\boldsymbol{\gamma}}\|}{\dot{d}_{0}-\kappa_{g}\|\dot{\boldsymbol{\gamma}}\|\left(1+d_{0}^{2}\right)} \in \mathbb{R} \cup\{\infty\} .
$$

The regularity condition (6) generalises to

$$
\left|\dot{d}_{0}-\kappa_{g}\|\dot{\boldsymbol{\gamma}}\|\left(1+d_{0}^{2}\right)\right|<\frac{\|\dot{\boldsymbol{\gamma}}\|}{w} .
$$

\section{Isometry of the Model}

In the previous section, we justify several geometric conditions for our model that ensure the isometric deformation of a strip. Now we aim at rigorously proving equivalence between these constraints and isometry. In order to differentiate between initial and deformed state, we indicate belongingness to the initial configuration with a superset ${ }^{\circ}$ for all variables. For simplicity, we assume that the initial state is planar here and generalise this statement later.

Proposition 3.1. Let $\phi^{\circ}$ be a planar surface described in the form (1), such that the director satisfies the regularity condition (7) and the scaling condition $\left\|\mathbf{d}^{\circ}-\left(\mathbf{d}^{\circ} \cdot \mathbf{t}^{\circ}\right) \mathbf{t}^{\circ}\right\| \equiv 1$.

Then $\boldsymbol{\phi}$ is an isometrically deformed state of $\boldsymbol{\phi}^{\circ}$ if and only if $\boldsymbol{\phi}$ is ruled and satisfies the following properties:

(a) The deformed state $\boldsymbol{\phi}$ is developable, i.e. $\operatorname{det}[\mathbf{t}, \mathbf{d}, \mathbf{d}] \equiv 0$.

(b) The geodesic curvature of the directrix is preserved, i.e. $\kappa_{g} \equiv \kappa_{g}^{\circ}$.

(c) There is no change in the width of the strip, i.e. $d_{1}^{2}+d_{2}^{2} \equiv 1$.

(d) There is no tensile strain along $\boldsymbol{\gamma}$, i.e. $\|\dot{\boldsymbol{\gamma}}\| \equiv\left\|\dot{\boldsymbol{\gamma}}^{\circ}\right\|$.

(e) There is no penetration of matter, i.e. $d_{0}$ satisfies (7). 
The subsequent proof establishes equivalence on the global level instead of proving necessity and sufficiency separately. Only for two minor claims, we treat both sides consecutively (indicated by " $\Leftarrow$ " or " $\Rightarrow$ ").

Proof. For the start, remember the Gaussian Theorema Egregium [14] after which Gaussian curvature is preserved under isometric deformations. As $\phi^{\circ}$ is planar, $\phi$ must be developable and therewith ruled if it is an isometrically deformed state. Therefore, $\phi$ may be parametrised in the form (1) with decomposed director (3) for both sides of the claim.

As we regard a flat reference configuration, $\gamma^{\circ}$ is a plane curve. We may construct a frame $\left(\mathbf{t}^{\circ}, \mathbf{m}_{1}^{\circ}, \mathbf{m}_{2}^{\circ}\right)$ from the curve tangent $\mathbf{t}^{\circ}$ and the constant plane normal $\mathbf{m}_{2}^{\circ}$. Note that this frame is a relatively parallel frame, but has only one non-zero curvature component $k_{1}^{\circ} \equiv: \kappa_{g}^{\circ}$, which is the (geodesic) curvature of $\boldsymbol{\gamma}^{\circ}$. This yields the representation

$$
\boldsymbol{\phi}^{\circ} \equiv \boldsymbol{\gamma}^{\circ}+v \mathbf{d}^{\circ}, \quad \mathbf{d}^{\circ} \equiv d_{0}^{\circ} \mathbf{t}^{\circ}+\mathbf{m}_{1}^{\circ} .
$$

We compute the expressions for the symmetric metric tensors $\mathbf{A}, \mathbf{A}^{\circ}$ and subtract them from each other. The entries of the resulting difference tensor $\Delta \mathbf{A}:=\mathbf{A}-\mathbf{A}^{\circ}$ may be written as polynomials in $v$ with coefficients $x_{11}, y_{1 \alpha}, z_{\alpha \beta}:[0, L] \rightarrow \mathbb{R}$ for $\alpha, \beta \in\{1,2\}$ defined via

$$
\Delta \mathbf{A}(u, v)=:\left[\begin{array}{rr}
x_{11}(u) v^{2}+y_{11}(u) v+z_{11}(u) & y_{12}(u) v+z_{12}(u) \\
y_{12}(u) v+z_{12}(u) & z_{22}(u)
\end{array}\right] .
$$

The deformation from $\boldsymbol{\phi}^{\circ}$ to $\boldsymbol{\phi}$ is isometric if and only if this difference tensor vanishes globally on $[0, L] \times[-w, w]$, especially for each $v \in[-w, w]$. This happens if and only if the six coefficient functions $x_{11}, y_{1 \alpha}, z_{\alpha \beta}$ are constantly zero. We consider one entry after the other in order to link them to the properties $(a)-(e)$ and immediately simplify the remaining coefficients.

"(d)" First consider $z_{11} \equiv\|\dot{\gamma}\|^{2}-\left\|\dot{\boldsymbol{\gamma}}^{\circ}\right\|^{2}$, which vanishes if and only if the curves $\boldsymbol{\gamma}, \boldsymbol{\gamma}^{\circ}$ have the same derivative norm.

“(e)" With this we compute $z_{12} \equiv\left(d_{0}-d_{0}^{\circ}\right)\|\dot{\boldsymbol{\gamma}}\|$, which vanishes if and only if $d_{0} \equiv d_{0}^{\circ}$. At first glance this might confuse, as the angle between rulings and tangent are supposed to change during arbitrary deformations. However, this indicates that a pair of coordinates $(u, v)$ describes different material points in reference and deformed configuration, respectively, if and only if the angles change.

Nevertheless, there is a valid parametrisation of the reference configuration for every $d_{0}^{\circ}$ that satisfies the edge-of-regression-condition. " $\Leftarrow$ " If property $(e)$ holds, we may choose $d_{0}^{\circ}$ matching $d_{0}$ in order to describe both configurations with the same parameter set. " $\Rightarrow$ " Otherwise, there would be a self-penetration of matter which cannot occur during an isometric deformation.

"(c)" We simplify $z_{22} \equiv d_{1}^{2}+d_{2}^{2}-1$ which enforces unit length of the projection of the director to the normal plane in deformed state if and only if the coefficient vanishes.

"(b)" The preservation of the geodesic curvature of the directrix is equivalent to

$$
y_{11} \equiv 2\|\dot{\gamma}\|\left(\kappa_{g}^{\circ}-k_{1} d_{1}-k_{2} d_{2}\right) \equiv 2\|\dot{\gamma}\|\left(\kappa_{g}^{\circ}-\kappa_{g}\right) \equiv 0 .
$$

“( a)" We end up with two remaining coefficients

$$
x_{11} \equiv \pi_{G}^{(1)^{2}}+\pi_{G}^{(2)^{2}}-\|\dot{\boldsymbol{\gamma}}\|^{2} d_{0}^{2} \kappa_{g}{ }^{2}, \quad y_{12} \equiv d_{1} \pi_{G}^{(1)}+d_{2} \pi_{G}^{(2)}-\|\dot{\boldsymbol{\gamma}}\| d_{0} \kappa_{g} .
$$

" $\Rightarrow$ " Assume that both $x_{11}$ and $y_{12}$ vanish completely (i.e. the deformation is isometric). Then we may solve the latter for $\|\dot{\boldsymbol{\gamma}}\| d_{0} \kappa_{g}^{\circ}$ and insert it in the former, which gives

$$
x_{11} \equiv\left(1-d_{1}^{2}\right) \pi_{G}^{(1)^{2}}+\left(1-d_{2}^{2}\right) \pi_{G}^{(2)^{2}}-2 d_{1} d_{2} \pi_{G}^{(1)} \pi_{G}^{(2)} .
$$


We insert $(c)$ and eventually get

$$
x_{11} \equiv d_{2}^{2} \pi_{G}^{(1)^{2}}+d_{1}^{2} \pi_{G}^{(2)^{2}}-2 d_{1} d_{2} \pi_{G}^{(1)} \pi_{G}^{(2)} \equiv\left(d_{1} \pi_{G}^{(2)}-d_{2} \pi_{G}^{(1)}\right)^{2} \equiv \operatorname{det}[\mathbf{t}, \mathbf{d}, \dot{\mathbf{d}}]^{2} \equiv-\kappa_{G} .
$$

Hence, $x_{11}$ vanishes only if the deformed configuration is developable.

" $\Leftarrow$ ” Now assume vice versa that $\phi$ is developable and thus

$$
\operatorname{det}[\mathbf{t}, \mathbf{d}, \dot{\mathbf{d}}] \equiv d_{1} \pi_{G}^{(2)}-d_{2} \pi_{G}^{(1)} \equiv 0 .
$$

Since (c), for each $u_{0} \in[0, L]$ we have $d_{1}\left(u_{0}\right) \neq 0$ or $d_{2}\left(u_{0}\right) \neq 0$ - without loss of generality let the former be true. Then we may solve

$$
0 \equiv \frac{\mathrm{d}}{\mathrm{d} u}(1) \equiv \frac{\mathrm{d}}{\mathrm{d} u}\left(d_{1}^{2}+d_{2}^{2}\right) \equiv d_{1} \dot{d}_{1}+d_{2} \dot{d}_{2}
$$

for $\dot{d}_{1}\left(u_{0}\right)$ and insert the result in (9). For clarity we omit the dependency in $u_{0}$, but use $=$ to indicate that the following statements hold in a single point:

$$
0=d_{1} \pi_{G}^{(2)}-d_{2} \pi_{G}^{(1)}=d_{1} \dot{d}_{2}+\frac{d_{2}^{2}}{d_{1}} \dot{d}_{2}+d_{0}\|\dot{\boldsymbol{\gamma}}\|\left(d_{1} k_{2}-d_{2} k_{1}\right) .
$$

We conclude that $d_{1}+\frac{d_{2}^{2}}{d_{1}}=\frac{1}{d_{1}}$ and solve for

$$
\dot{d}_{2}=-d_{1} d_{0}\|\dot{\boldsymbol{\gamma}}\|\left(d_{1} k_{2}-d_{2} k_{1}\right) .
$$

We plug this and the corresponding term for $\dot{d}_{1}$ into $y_{12}$ and get

$$
y_{12} \equiv \underbrace{d_{1} \dot{d}_{1}+d_{2} \dot{d}_{2}}_{\equiv 0}+\underbrace{d_{0}\|\dot{\boldsymbol{\gamma}}\|\left(d_{1} k_{1}+d_{2} k_{2}\right)-d_{0}\|\dot{\boldsymbol{\gamma}}\| \kappa_{g}}_{\equiv 0} \equiv 0,
$$

which holds globally due to the arbitrary choice of $u_{0}$. As we established $y_{12} \equiv 0$, we may use (8) for $x_{11}$ which vanishes by assumption.

For generalisation, consider the case of a non-planar but still developable reference configuration $\boldsymbol{\phi}^{\circ}$. By the definition of developability, there exists a planar state $\boldsymbol{\phi}^{p}$ that is an isometric deformation of $\phi^{\circ}$. The planar state is obviously developable and needs to fulfil

$$
1 \equiv \operatorname{det} \mathbf{A}^{\circ}(\cdot, 0) \equiv \operatorname{det} \mathbf{A}^{p}(\cdot, 0) \equiv d_{1}^{p 2}+d_{2}^{p 2} .
$$

Hence, we may interchange the roles of $\phi^{\circ}$ and $\phi^{p}$ and apply Proposition 3.1 on them and, then again, we may employ Proposition 3.1 for the transformation between $\phi^{p}$ and $\phi$.

Corollary 3.2. Let $\phi^{\circ}$ be a developable surface described in the form (1), such that the director satisfies the regularity condition (7) and the scaling condition $\left\|\mathbf{d}^{\circ}-\left(\mathbf{d}^{\circ} \cdot \mathbf{t}^{\circ}\right) \mathbf{t}^{\circ}\right\| \equiv 1$.

Then $\boldsymbol{\phi}$ is an isometrically deformed state of $\boldsymbol{\phi}^{\circ}$ if and only if $\boldsymbol{\phi}$ is ruled and satisfies the following properties:

(a) The deformed state $\boldsymbol{\phi}$ is developable, i.e. $\operatorname{det}[\mathbf{t}, \mathbf{d}, \dot{\mathbf{d}}] \equiv 0$.

(b) The geodesic curvature of the directrix is preserved, i.e. $\kappa_{g} \equiv \kappa_{g}^{\circ}$.

(c) There is no change in the width of the strip, i.e. $d_{1}^{2}+d_{2}^{2} \equiv 1$.

(d) There is no tensile stress along $\boldsymbol{\gamma}$, i.e. $\|\dot{\boldsymbol{\gamma}}\| \equiv\left\|\dot{\boldsymbol{\gamma}}^{\circ}\right\|$.

(e) There is no penetration of matter, i.e. $d_{0}$ satisfies (7). 


\section{Energy Functional}

As we established in the previous section, the conditions in Corollary 3.2 yield geometric constraints for isometric deformations of developable bands. Thus, we may compute the static equilibrium state of a strip under these requirements and given boundary conditions by minimising the stored energy. As we already outlined, there is no membrane deformation present such that this energy functional only consists of the bending part.

For the sake of simplicity, we consider a rectangular strip with geodesic base line $\boldsymbol{\gamma}$ parametrised by arc-length, and a flat reference configuration. We compute the mean curvature $H$ of a ruled surface (1) and simplify the resulting expression via (5). This results in

$$
H(\cdot, v) \equiv \frac{\left(d_{1} k_{2}-d_{2} k_{1}\right)\left(d_{0}^{2}+1\right)}{1+v d_{0}^{\prime}} .
$$

Note that the denominator of $H$ matches exactly the transformation factor for the area element $\mathrm{d} A=\left(1+v d_{0}^{\prime}\right) \mathrm{d} s \mathrm{~d} v$. Hence, the bending energy of a uniformly isotropic reads

$$
\begin{aligned}
\Xi(\phi)=\frac{D}{2} \iint_{\boldsymbol{\phi}} H^{2} \mathrm{~d} A & =\frac{D}{2} \int_{0}^{L}\left(d_{1} k_{2}-d_{2} k_{1}\right)^{2}\left(d_{0}^{2}+1\right)^{2} \int_{-w}^{w} \frac{1}{1+v d_{0}^{\prime}} \mathrm{d} v \mathrm{~d} s \\
& =D w \int_{0}^{L}\left(d_{1} k_{2}-d_{2} k_{1}\right)^{2}\left(d_{0}^{2}+1\right)^{2} V\left(w d_{0}^{\prime}\right) \mathrm{d} s,
\end{aligned}
$$

where, $D=\frac{2 t^{3} E}{3\left(1-v^{2}\right)}$ denotes the flexural rigidity of the material with Young's modulus $E$ and Poisson ration $v$ and

$$
V\left(w d_{0}^{\prime}\right)=\frac{1}{w d_{0}^{\prime}} \log \left(\frac{1+w d_{0}^{\prime}}{1-w d_{0}^{\prime}}\right)=1+\mathscr{O}\left(\left(w d_{0}^{\prime}\right)^{2}\right)
$$

gives the small width approximation term which may be neglected under linearisation about an infinitely narrow band [5]. Note that non-rectangular strips yield the same energy functional (10) with an additional additive term in the argument of $V$.

\section{Numerical Model and Implementation}

In order to compute the stable static equilibrium of a rectangular strip under specified boundary conditions, we minimise the energy functional (10) regarding the geometric constraints 3.2(a)-(d) and the simplified regularity condition 6 . The base curve $\gamma$ and the director field $\mathbf{d}$ constitute the degrees of freedom, where latter is represented by the coefficient functions $d_{i}$.

Requiring 3.2(c) introduces an angle $\sigma$ such that $d_{1} \equiv-\sin \sigma$ and $d_{2} \equiv \cos \sigma$. This angle also occurs as polar coordinate of the curvatures $\left(k_{1}, k_{2}\right):[0, L] \rightarrow \mathbb{R}^{2}$ and between relatively parallel and Frenet frame, wherever latter exists. Thereby, 3.2(c) is taken into account explicitly.

A penalty approach addresses the remaining constraints

$$
\begin{aligned}
\min _{\boldsymbol{\gamma}, d_{0}, \sigma} & \Xi+c_{1} \Psi+c_{2} \Theta+c_{3} \Omega \\
\Psi & =\int_{0}^{L} \operatorname{det}[\dot{\boldsymbol{\gamma}}, \mathbf{d}, \dot{\mathbf{d}}]^{2} \mathrm{~d} u \\
\Theta & =\int_{0}^{L} \kappa_{g}^{2} \mathrm{~d} u, \\
\Omega & =\int_{0}^{L}(\|\dot{\gamma}\|-1)^{2} \mathrm{~d} u
\end{aligned}
$$

with penalty weights $c_{i}>0$. In this formulation, $c_{1} \rightarrow \infty$ ensures developability, $c_{2} \rightarrow \infty$ maintains the geodesic property of $\boldsymbol{\gamma}$ and $c_{3} \rightarrow \infty$ enforces the inextensibility of the base curve. 
As this model requires at least $\mathscr{C}^{2}$-smoothness (almost everywhere), isogeometric curves are predestinated candidates for discretisation of $\gamma, d_{0}$ and $\sigma$. Given a polynomial degree $p$ and the number of desired control points $n+1$, a clamped knot vector $U=\left[u_{0}, \ldots, u_{n+p+1}\right]$ with

$$
0=u_{0}=\ldots u_{p}<u_{p+1} \leq \cdots \leq u_{n}<u_{n+1}=\cdots=u_{n+p+1}=L
$$

defines the family of B-Spline basis functions $\left\{N_{i, p}:[0, L] \rightarrow[0,1] \mid i=0, \ldots, n\right\}$. These are constructed recursively by

$$
\begin{aligned}
& N_{i, 0}(u)= \begin{cases}1, & , \text { if } u \in\left[u_{i}, u_{i}+1\right] \\
0, & \text { otherwise }\end{cases} \\
& N_{i, p}(u)=\frac{u-u_{i}}{u_{i+p}-u_{i}} N_{i, p-1}(u)+\frac{u_{i+p+1}-u}{u_{i+p+1}-u_{i+1}} N_{i+1, p-1} .
\end{aligned}
$$

A B-Spline curve $\boldsymbol{\beta}$ in $q$ dimensions then reads

$$
\boldsymbol{\beta}:[0, L] \rightarrow \mathbb{R}^{q}, u \mapsto \sum_{i=0}^{n} N_{i, p}(u) \mathbf{p}_{i},
$$

where $\mathbf{p}_{i} \in \mathbb{R}^{q}$ are the control points, which become the degrees of freedom for our approach. For details on the properties of B-Spline basis functions, NURBS, and related curves confer [15].

For minimisation, we utilise the interior point optimiser IPopt [9] on the functional 11, where the boundary conditions are treated as constraints. The automatic differentiation library autodiff [16] computes the derivatives for gradients and Hessian matrices. Theoretical background may be looked up in [17].

\section{Results and Conclusion}

We choose three benchmarks to apply the model described in the previous section on. For each case, the boundary conditions and a flat reference geometry pose the input such that length and width of the plane strip coincide with the $\mathrm{x}$ - and $\mathrm{y}$-axis, respectively.

For the first example, the start $(u=0)$ remains completely fixed, whereas the end $(u=L)$ is dragged towards the start and the tangent direction in the end point is flipped upwards by $\frac{\pi}{4}$. Under these boundary conditions, the problem reduces to two dimensions where the director is transported parallelly along the plane curve. Figure 3 illustrates the equilibrium state.

The boundary conditions of the second use depicted in 4 case enforce a spatial base curve. As before, the start is kept fixed whereas the second end is translated in both negative $\mathrm{x}$ - and positive $y$-direction and the tangent remains fixed. Additionally, we specify a boundary condition for the director at the second end.

The third example displayed in Figure 5 illustrates the extension to curved reference directrices. Instead of a purely rectangular reference state, we choose a quarter of a two-dimensional torus with radius 4 . Hence, the (geodesic) curvature of the base curve is constantly $\kappa_{g}^{\circ} \equiv \frac{1}{4}$ and needs to be preserved in the deformed state. Therefore, the penalty term $\Theta$ in (11b) is updated to yield preservation of curvature as $\int_{0}^{L}\left(\kappa_{g}-\kappa_{g}^{\circ}\right)^{2} \mathrm{~d} u$. We then drag the second end $(u=L)$ both upward and in opposite tangent direction.

Although all example benchmarks compute suiting equilibrium configurations, the computations involve high iteration numbers with small steps that indicate susceptibility to slow convergence. The condition numbers of the Hessian matrix within the last iteration for the three examples read $9.5 \cdot 10^{17}, 1.1 \cdot 10^{18}, 1.5 \cdot 10^{17}$ respectively. Hence, the solution process requires numerical improvement in order to achieve real time applicability.

Furthermore, an ordinary differential equation related to the relatively parallel transport of normal vectors may substitute automatic differentiation. In order to properly model FFCs, the bending energy of section 4 requires incorporation of an anisotropic material law. 


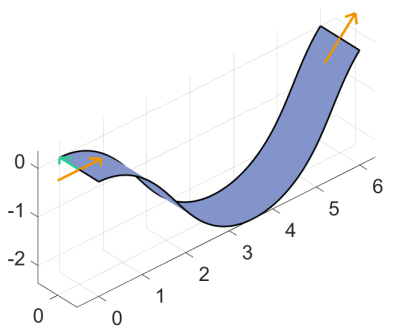

Figure 3: Equilibrium configuration under planar boundary conditions.

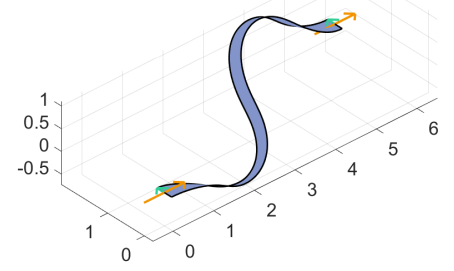

Figure 4: Example with nonplanar base curve.

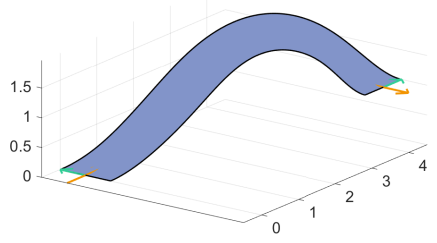

Figure 5: Example with curved reference directrix.

\section{REFERENCES}

[1] Chapelle, D., Bathe, K.J.: The Finite Element Analysis of Shells - Fundamentals: Fundamentals. Computational Fluid and Solid Mechanics. Springer-Verlag Berlin Heidelberg, Berlin, Heidelberg (2011) ISBN: 978-3-642-16408-8.

[2] Bischoff, M., Bletzinger, K.U., Wall, W.A., Ramm, E.: Models and finite elements for thin-walled structures. In: Encyclopedia of computational mechanics. Volume 172. Wiley, Chichester (2004) ISBN:0470846992.

[3] Fosdick, R., Fried, E., eds.: The Mechanics of Ribbons and Möbius Bands. 1st edn. Springer Netherlands, Dordrecht and s.l. (2016) ISBN:978-94-017-7300-3.

[4] Sadowsky, M.: Ein elementarer Beweis für die Existenz eines abwickelbaren Möbiusschen Bandes und Zurückführung des geometrischen Problems auf ein Variationsproblem. Sitzungsberichte der Preussischen Akademie der Wissenschaften (1930)

[5] Wunderlich, W.: Über ein abwickelbares Möbiusband. Monatshefte für Mathematik 66(3) (1962) 276-289 doi:10.1007/BF01299052.

[6] Starostin, E.L., van der Heijden, G.H.M.: Equilibrium shapes with stress localisation for inextensible elastic möbius and other strips. Journal of Elasticity 119(1) (2015) 67-112 doi:10.1007/s10659-014-9495-0.

[7] Dias, M.A., Audoly, B.: "Wunderlich, meet Kirchhoff": A general and unified description of elastic ribbons and thin rods. Journal of Elasticity 119(1-2) (2015) 49-66 doi:10.1007/s10659-014-9487-0.

[8] Bishop, R.L.: There is more than one way to frame a curve. The American Mathematical Monthly 82(3) (1975) 246-251 doi:10.2307/2319846.

[9] Wächter, A., Biegler, L.T.: On the implementation of an interior-point filter line-search algorithm for large-scale nonlinear programming. Mathematical Programming 106(1) (2006) 25-57 doi:10.1007/s10107-004-0559-y.

[10] Struik, D.J.: Lectures on classical differential geometry. 2nd edn. Dover Publications, Mineola, NY, USA (1988) ISBN:0486656098.

[11] Krejčiř́ik, D., Šediváková, H.: The effective hamiltonian in curved quantum waveguides under mild regularity assumptions. Reviews in Mathematical Physics 24(07) (2012) doi:10.1142/S0129055X12500183.

[12] Izumiya, S., Takeuchi, N.: New special curves and developable surfaces. Turkish Journal of Mathematics 28 (2004) 153-163 doi: 10.14943/83700. 
[13] do Carmo, M.P.: Differential geometry of curves and surfaces. Prentice-Hall, Englewood Cliffs, New Jersey, USA (1976) ISBN:0132125897.

[14] Gauss, C.F.: Allgemeine Flächentheorie (disquisitiones generales circa superficies curvas). Engelmann, Leipzig, Germany (1889) doi:10.24355/DBBS.084-201303011402-0.

[15] Piegl, L., Tiller, W.: The NURBS Book. Springer, Berlin, Heidelberg (1995) ISBN:978-3642-97387-1.

[16] Leal, A.: Autodiff, a modern, fast and expressive C++ library for automatic differentiation. (2018) url: https://autodiff.github.io.

[17] Griewank, A., Walther, A.: Evaluating derivatives: Principles and techniques of algorithmic differentiation. 2nd edn. Society for Industrial and Applied Mathematics, Philadelphia, USA (2008) ISBN:978-0-89871-659-7. 